\title{
The Fifth International Conference on Dietary Assessment Methods, Chiang- Rai, Thailand, 26-29 January 2003
}

The goals of the conference were to address methodological issues of dietary assessment in diverse populations and cultural mixes, and to enhance understanding on changes in dietary pattern and the challenges facing the application and improvement of dietary assessment methods in a multicultural environment. About 400 participants from 44 countries attended the conference, which was opened by Her Royal Highness Mahachakri Sirindhorn.

The conference was based on the need to:

- investigate diet-health relationships

- identify population groups at greatest risk

- assess exposure to foods and dietary constituents related to chronic disease risks

- formulate food and nutrition policies to reduce those risks

- select appropriate nutrition intervention programmes

- monitor and evaluate the effectiveness of these programmes

- address issues of food security at various levels

- estimate exposure to contaminants

- determine group compliance with dietary guidelines.

Cross-cultural studies of populations encompassing a broad range of dietary intakes were a major focus of this conference. Despite methodological difficulties, these international comparison studies are essential in understanding dietary assessment issues in a multicultural world.

An important issue for discussion during the conference was the validity of dietary data collected through food frequency questionnaires (FFQs) in large epidemiological investigations. Professor Walter Willet, epidemiologist, and user of FFQs in several large-scale nutritional epidemiological studies, at Harvard School of Public Health, USA, and Professor Victor Kipnis, statistician at the National Cancer Institute, Bethesda, USA, met in a debate to present the pros and cons of using FFQs in epidemiological investigations. Professor Willett reported that errors in dietary assessments are inevitable, although they can be reduced by the careful design of questionnaires, iterative evaluation and enhancement of these methods, and repeated measurements. Professor Kipnis demonstrated the effects of uncertainty in data collection in studies, with a focus on correlations between dietary factors and the incidence of disease. He also illustrated the problems with overestimating and underestimating as effects of estimating dietary data incorrectly. The question was raised as to whether people in the future no longer will collect dietary data by FFQs, even if this is considered to be a rational way of collecting data, but rather develop methods that will describe individuals' daily intake by dietary interviews, computerized dietary recalls or via the Internet. This development of methods was proposed as a possible topic at the next conference in Scandinavia.

At the different poster sessions throughout the conference a great number of validation studies, in combination with biological markers, were presented. In an early morning session issues regarding how to use the new American and Canadian dietary reference intakes were covered. The session was held by Suzanne Murphy from the Cancer Research Institute of Hawaii, and Alicia Carriquiry, a wellknown statistician from Iowa State University, USA. They are both members of the Subcommittee on interpretation and uses of dietary reference intakes, Food and Nutrition Board, Institute of Medicine, USA.

The Sixth Conference on Dietary Assessment Methods will be held in Copenhagen in 2006, hosted by the University of Copenhagen and the University of Gothenburg.

Margaretha Nydahl and Lillemor Abrahamson

Department of Domestic Sciences, Uppsala University, Dag Hammarskjöldsväg 21, SE-752 37 Uppsala, Sweden

E-mail: Margaretha.Nydahl@ihv.uu.se 